\title{
VELOCIDAD DEL SONIDO Y PÉRDIDAS DE ENERGÍA EN TUBOS CILÍNDRICOS
}

\section{Speed of sound and energy losses in cylindri cal tubes}

DOI https://doi.org/10.36411/AEF.1.2.10

Francisco Echegorri

ORCID https://orcid.org/0000-0002-6727-0924

Consejo de Formación en Educación, Instituto de Profesores Artigas, Montevideo, Uruguay

francechegorri@gmail.com

Recibido: 05 de noviembre de 2019

Aceptado: 23 de diciembre de 2019

\section{Resumen}

Es bien conocido que las ondas acústicas en el aire son ondas no dispersivas, propagándose a la misma velocidad independientemente de la frecuencia de la perturbación que las generó. En cambio, las ondas acústicas que se propagan en el interior de un tubo delgado y largo forman un patrón de ondas estacionarias constituido por la superposición de muchas ondas de diferentes frecuencias. Cada una de estas ondas viajeras se propaga con una velocidad diferente debido al fenómeno de dispersión producido por las pérdidas de energía viscosa y térmica en las paredes del tubo. Aquí se analiza dicho fenómeno y se estudia experimentalmente con materiales de bajo costo, haciendo uso de las potencialidades de los teléfonos inteligentes. Se determina también un procedimiento para hallar la velocidad del sonido en el aire libre.

Palabras claveVelocidad del sonido, resonancia acústica, pérdidas de energía en tubos cilíndricos, guía de ondas acústicas, experimentos de bajo costo, experimentos con teléfonos inteligentes.

\begin{abstract}
It is well known that the acoustic waves in the air are non-dispersive waves, propagating at the same speed regardless of the frequency of the disturbance that generated them. On the other hand, the acoustic waves that propagate inside a thin and long tube form a standing wave pattern constituted by the superposition of many waves of different frequencies. Each of these traveling waves travels at a different speed due to the dispersion phenomenon produced by the viscous and thermal energy losses in the walls of the tube. Here the phenomenon is analyzed and experimentally studied with low-cost materials, making use of the potential of smartphones. A procedure is also determined to find the speed of sound in the open air.

Keywords: Speed of sound, acoustic resonance, wall loss in cylindrical tubes, acoustic wave guide, low cost experiments, experiments with smart phones.
\end{abstract}

\section{Introducción}

En la enseñanza de la Física de los cursos de formación docente o de las carreras de ciencias e ingeniería, el laboratorio constituye un elemento muy importante dentro del aprendizaje. En años recientes el uso de las TIC (Gil, 2014) permite realizar usando las potencialidades de los teléfonos inteligentes un sinnúmero de experiencias de bajo costo usando los sensores físicos incorporados a los mismos: acelerómetros, barómetros, sensores Hall, sensores de luz, giroscopios, termómetros, micrófonos, etc. El micrófono incorporado al mismo y el software de análisis dinámico de una señal acústica (FFT en tiempo real) permite medir fácilmente las frecuencias de resonancia de las ondas estacionarias presentes en un tubo. Es usual en los cursos terciarios realizar experiencias para medir la velocidad del sonido en el aire libre usando el fenómeno de 
resonancia(Práctica de Ondas Mecánicas). Sin importar el material usado para registrar las frecuencias de dichas ondas y la forma en que son generadas (usando generadores de funciones, parlantes, osciloscopios, amplificador lock-in, etc.) la mayoría de los docentes que alguna vez han realizado la experiencia encuentran una pequeña discrepancia entre la frecuencia del primer armónico medida y la predicción calculada a partir del valor de la velocidad del sonido en el aire libre a esa temperatura y humedad. En una experiencia realizada usando un tubo de $1,202 \mathrm{~m}$ de largo y $0,970 \mathrm{~cm}$ de radio (con una velocidad del sonido calculada a partir de la temperatura y humedad del aire de $346,44 \mathrm{~m} / \mathrm{s}$ ) se realizó una experiencia muy cuidada en donde se midieron las frecuencias de resonancia de un tubo abierto-cerrado. Los resultados experimentales se resumen en la tabla 1 :

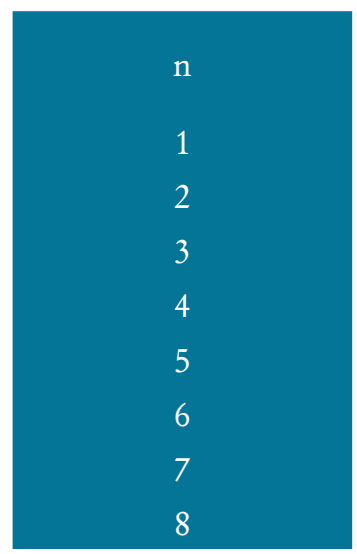

$\left(f_{n \exp } \pm 0,135\right)(\mathrm{Hz})$
70,27
212,72
355,68
498,38
641,50
784,37
927,45
1070,77

$\begin{array}{cc}\begin{array}{c}f_{\text {n teo }} \\ (H z)\end{array} & \text { Corrimiento }(\%) \\ 71,70 & -2,0 \\ 215,10 & -1,1 \\ 358,50 & -0,79 \\ 501,90 & -0,70 \\ 645,30 & -0,59 \\ 788,70 & -0,55 \\ 932,10 & -0,50 \\ 1075,50 & -0,44\end{array}$

Tabla 1. Medida de las distintas frecuencias de resonancia en un tubo abierto - cerrado en función del número de modo normal n. La frecuencia teórica se calculó suponiendo conocida la velocidad del sonido en el interior del tubo.

Es evidente observando el corrimiento porcentual que este presenta un comportamiento sistemático (no aleatorio), disminuyendo gradualmente para las frecuencias de los armónicos superiores.

En general se atribuye esa pequeña discrepancia en el primer valor de la frecuencia (aunque está presente en todos los modos) a errores experimentales asociados al equipamiento o a los procedimientos usados para realizar la experiencia. En ningún caso se asume que las ondas sonoras en el interior del tubo son dispersivas. Sin embargo, mostraremos aquí que usando equipamiento modesto es posible medir la variación de la velocidad del sonido en el interior del tubo para las distintas frecuencias de los armónicos y verificar el modelo teórico que muestra el fenómeno de dispersión (variación de la velocidad de fase en función de la frecuencia) en el interior del tubo.

Las pérdidas de energía y su influencia en las frecuencias de resonancia para tubos abiertos y cerrados son de suma importancia en la construcción de órganos y en los instrumentos de viento (Fletcher,1983) (su longitud y diámetro quedan largamente determinados por dichos efectos) Dichos efectos fueron estudiados en el siglo XIX por Helmholtz (Helmholtz,1863) Kirchhoff (Kirchhoff,1868) y Rayleigh (Rayleigh,1896) ${ }^{1}$

${ }^{1}$ La teoría completa de la propagación de ondas sonoras en tubos cilíndricos fue resumida por H. Tijdeman [7] 


\section{Teoría}

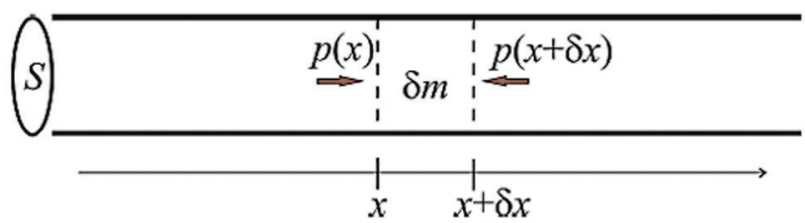

Fig. 1 Ondas acústicas propagándose por un tubo de sección uniforme.

Las ondas sonoras en el aire se deben a pequeñas variaciones de la presión del aire respecto a su presión en el equilibrio denominada presión acústica. Dichas variaciones se propagan como una onda en el medio (ver Fig. 1). Un tubo cilíndrico de longitud finita puede propagar en general ondas acústicas planas en la dirección del eje del cilindro si la frecuencia de la perturbación acústica es menor a la primer frecuencia de corte en ondas guiadas (Linsler,1995) ${ }^{2}$

$$
f_{c}=\frac{1,84 c}{2 \pi a}(1)
$$

siendoc la velocidad del sonido y a es el radio del tubo. El movimiento de las partículas del aire es colineal con la dirección de propagación de las ondas, por lo cual estas son ondas longitudinales. Según Kinsler la presión acústica verifica para las ondas de pequeña amplitud en una dimensión la ecuación

$$
\frac{\partial^{2} p}{\partial t^{2}}=c^{2} \frac{\partial^{2} p}{\partial x^{2}}(2)
$$

Donde c es la velocidad del sonido. La velocidad del sonido está determinada por (3), donde $\mathrm{B}$ es el módulo de compresibilidad adiabático ${ }^{3}$ y es la densidad del aire.

$$
c=\sqrt{\frac{B}{\rho}}(3)
$$

En la aproximación del aire como gas perfecto, la velocidad del sonido está dada por:

$$
c=\sqrt{\frac{\gamma R T}{\bar{M}}}(4)
$$

\footnotetext{
${ }^{2} \mathrm{En}$ todos los experimentos realizados las frecuencias medidas en los tubos eran menores a la frecuencia de corte (1), por ejemplo, en la experiencia resumida en la tabla 1, la frecuencia de corte fue $\mathrm{fc}=10,46 \mathrm{kHz}$ y la frecuencia del octavo armónico, tenía el valor de $\mathrm{f}=1070,77 \mathrm{~Hz}$
}

${ }^{3} \mathrm{En}$ el caso de las ondas sonoras en un gas las variaciones de presión son tan rápidas que las compresiones y descompresiones pueden ser bien descriptas por un proceso adiabático. 
Siendo $\gamma=\frac{C_{p}}{C}$ la razón de los calores específicos a presión y volumen constantes, $\mathrm{T}$ es la temperatura en Kelvin y es la masa molecular media del aire.

$\mathrm{A} 0^{\circ} \mathrm{C}$, y a una temperatura $\mathrm{t}$ en grados centígrados se aproxima por: [8]

$$
c=331,4+0,60 t(5)
$$

Las frecuencias de resonancia para un tubo de largo L y radio a cerrado en un extremo y abierto en el otro está dada por [8]:

$$
f=\frac{(2 n-1) c_{p}}{4 L_{e f}} n=1,2,3 \ldots(6)
$$

Siendo $c_{\mathrm{p}}$ es la velocidad de fase de las ondas acústicas en el interior del tubo definida a

$$
\text { partir de: } c_{p}=\frac{\omega}{k}(7)
$$

Siendo $\omega$ la frecuencia angular de las ondas y $\mathrm{k}$ el número de onda.

En la ecuación (8), $L_{\text {ef }}$ es la longitud efectiva del tubo, dicha longitud es mayor al largo del tubo, debido a que en el extremo abierto, la onda acústica no presenta un nodo de presión, sino que este está ubicado a una distancia 0,61veces el radio del tubo, ya que el tubo radia energía a su entorno ${ }^{4}$. El valor de la longitud efectiva está dado por: (Kinsler,1995).

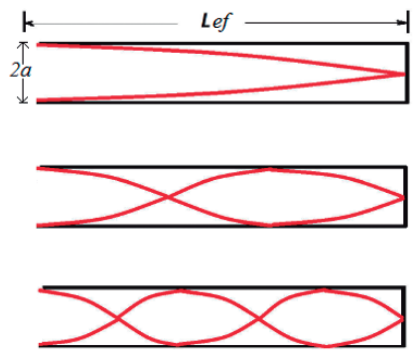

Fig. 2. Distribución de desplazamientos de las partículas de aire (representadas transversalmente) para los tres primeros modos normales para un tubo abierto-cerrado.

A partir de lasecuaciones (6) y (8) es posible medir la velocidad de fase de las ondas acústicas (midiendo las frecuencias de resonancia) para las distintas frecuencias de los modos normales de las ondas estacionarias (ver Fig. 2) y conociendo el largo del tubo.

\section{II.1 Pérdidas en las paredes del tubo}

Cuando las ondas acústicas planas se propagan en el interior de un tubo se atenúan debido a las pérdidas de energía por fricción y por intercambio de calor con las paredes del mismo ${ }^{5}$.

${ }^{4}$ La corrección de extremo libre es en este caso para un tubo sin pestaña, es decir un tubo que no presenta un reborde transversal al tubo.

- 44. $\quad{ }^{5}$ Las ondas acústicas planas se propagan en el interior de un tubo, para frecuencias menores a la frecuencia de corte, ecuación (1), para frecuencias mayores, las ondas se atenúan exponencialmente con la distancia de propagación, formando ondas evanescentes [10]. 
Las consideraciones detalladas del fenómeno de pérdidas en las paredes y sus efectos son complicadas pero el fenómeno básico y sus resultados son sencillos y los discutiremos aquí.

En "On the Propagation of Sound Waves in a Cylindrical Conduit" A. H Benade (Benade, 1968) analiza dichos resultados. Las paredes del tubo ejercen una fuerza viscosa que produce una aceleración del aire y que tiene asociada una impedancia. La magnitud relativa de la resistencia viscosa depende del espesor de la capa límite viscosa, a su vez este es proporcional a la raíz cuadrada de la viscosidad cinemática dividida por la frecuencia angular. Un parámetro conveniente es usar una magnitud adimensionada $r_{\mathrm{v}}$ (Boundary Layer Thickness Ratio (Rossing, 1994) definido como la relación entre el radio del tubo y el espesor de la capa límite viscosa, espesor $\delta_{v}$ que depende de la frecuencia angular $\omega$, de las ondas armónicas que se propagan en el interior del tubo:

$$
\begin{array}{r}
\delta_{v}=\left(\frac{\eta}{\rho \omega}\right)^{1 / 2}(9) \\
r_{v}=\frac{a}{\delta_{v}}=a\left(\frac{\omega}{v}\right)^{1 / 2}(10)
\end{array}
$$

Siendo $v=\frac{\eta}{\rho}$ la viscosidad cinemática, la viscosidad dinámica y la densidad del aire.

Del mismo modo, el intercambio térmico entre el aire y las paredes agrega una resistencia que también acelera el aire en las cercanías de la pared. La magnitud de esta pérdida depende de la relación del radio de la tubería al espesor de la capa límite térmico:

$$
\delta_{t}=\left(\frac{\kappa}{\omega \rho C_{p}}\right)^{1 / 2}
$$

Donde es la conductividad térmica del aire y es el calor específico del aire a presión constante. Es conveniente en este caso utilizar el parámetro adimensionado $r_{t}$ definido análogamente como habíamos hecho anteriormente como el cociente entre el radio del tubo y el espesor de la capa límite térmico:

$$
r_{t}=\frac{a}{\delta_{t}}=a\left(\frac{\omega \rho C_{p}}{\kappa}\right)^{1 / 2}
$$

La velocidad de fase $c_{p}$ en el interior de un tubo está dada con buena aproximación (para valores de $r_{v}>10$ y resulta adecuada para $r_{v}>3$ ) por (Rossing, 1994):

$$
c_{p} \approx \mathcal{c}\left[1-\frac{1}{r_{v} \sqrt{2}}-\frac{(\gamma-1)}{r_{i} \sqrt{2}}\right](13)
$$


La dependencia de los parámetros adimensionados $r_{\mathrm{v}}$ y $r_{t}$ (a partir de la dependencia con la temperatura, de la conductividad térmica, el calor específico la viscosidad y la densidad) vienen dados por [10]:

$$
\begin{aligned}
& r_{v} \approx 632.8 a f^{1 / 2}[1-0.0029(t-26,85)](14) \\
& r_{t} \approx 532.2 a f^{1 / 2}[1-0.0031(t-26,85)](15)
\end{aligned}
$$

Siendo a el radio del tubo en metros, $f$ la frecuencia en Hertz de las ondas armónicas y t la temperatura del aire en grados centígrados. Obtenemos para la velocidad de fase de las ondas acústicas en el interior de un tubo a partir de las ecuaciones (13), (14) y (15):

$$
c_{p} \approx c\left[1-\frac{1,65 \times 10^{-3}}{a f^{1 / 2}}-\frac{4,9 \times 10^{-6}(t-26.85)}{a f^{1 / 2}}\right](16)
$$

Siendo c la velocidad del sonido en el aire. La ecuación (16) muestra claramente que las ondas acústicas en el interior de un tubo delgado y largo son ondas dispersivas $^{6}$, siendo la velocidad de fase una función de la frecuencia de las ondas que se propagan en su interior.

\section{El experimento}

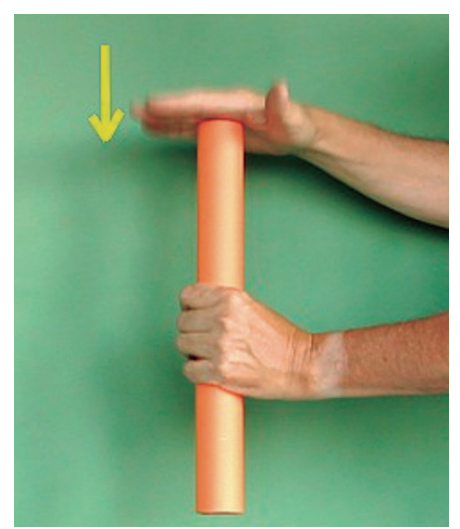

Figura 3. Tubo golpeado manteniendo la mano.

Se utilizaron para la experiencia 4 tubos de PVC de paredes delgadas (para minimizar el efecto de pestaña) de diferentes longitudes que van desde $0,638 \mathrm{~m}$ hasta $1,880 \mathrm{~m}$ de largo y los radios van desde $1,670 \mathrm{~cm}$ hasta $2,805 \mathrm{~cm}$ y un tubo de aluminio de $1,202 \mathrm{~m}$ de largo y $0,970 \mathrm{~cm}$ de radio. Los diámetros fueron medidos usando calibre con una apreciación de $0,05 \mathrm{~mm}$. La temperatura se midió con termómetro digital con una apreciación de $0,1^{\circ} \mathrm{C}$ y la humedad con una apreciación del $1 \%$.

${ }^{6}$ El segundo y el tercer factor de la ecuación (16) depende del producto del radio a del tubo y de la frecuencia elevada a la $1 / 2$, para tubos delgados, a es pequeño, las frecuencias de resonancia son también relativamente pequeñas, para tubos largos, ecuaciones (6) y (8), haciendo que dichos factores, disminuyan la velocidad de fase de las ondas acústicas en el interior del tubo, para todos los armónicos. El efecto es más importante para el primer armónico, y disminuye gradualmente al aumentar la frecuencia. 
El procedimiento utilizado consiste como muestra la figura 3 en golpear el tubo con la mano, y al finalizar el golpe mantenerla de modo que dicho extremo sea cerrado. Es conveniente colocar el micrófono del teléfono inteligente cerca del otro extremo del tubo. Luego de golpear el tubo, pausar la aplicación para registrar la FFT (transformada rápida de Fourier) cuando el gráfico en tiempo real presente una serie de picos bien definidos (ver figura 4). Luego moviendo el cursor, registrar los valores de las frecuencias de los distintos armónicos. Para registrar las frecuencias de los diferentes modos normales se utilizó la aplicación Spectrum Analyser (Spectrum Analyser) que permite realizar en tiempo real la FFT de la señal acústica registrada con el micrófono del teléfono inteligente.

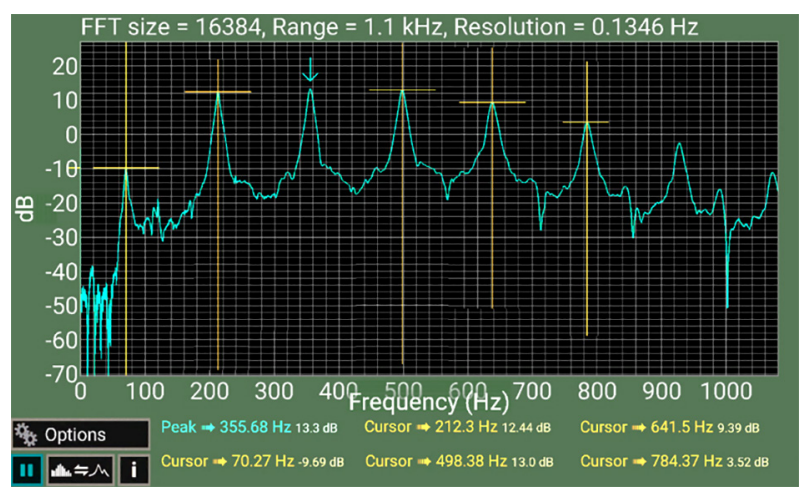

Figura 4. Ventana gráfica de la aplicación SpectrumAnalyser, donde se observan las frecuencias de resonancia de un tubo abierto-cerrado de $1,20 \mathrm{~m}$ de largo y $0,970 \mathrm{~cm}$ de radio. Fotomontaje con las frecuencias principales de resonancia. La amplitud del primer y segundo armónico es menor a la del tercero debido a que la respuesta del micrófono no es plana en este rango de frecuencias.

\section{III.1 Resultados experimentales}

La experiencia realizada en este caso queda resumida en la tabla 2 y en el gráfico de la figura 5. Se utilizó un tubo de aluminio de 1,202(1) m y un radio de $0,970(25) \mathrm{cm}$. La primera columna de la tabla 2, indica el número de armónico, para los distintos modos normales de las ondas en el interior del tubo.

La segunda columna registra las frecuencias medidas ${ }^{7}$ experimentalmente, la tercera columna es la velocidad de fase calculada a partir de las frecuencias experimentales usando las ecuaciones (6) y (8).

Su incertidumbre se calculó propagando incertidumbres en la ecuación (6). La velocidad de fase teórica (columna 4) se calculó a partir de la ecuación (16), para ello se determinó la velocidad del sonido en base a una temperatura de $23,8^{\circ} \mathrm{C}$ y una humedad relativa del 54\% usando la aplicación en línea en la página: www. sengpielaudio.com/calculator-airpressure.htm[12]

aplicación basada en un modelo para el aire húmedo [13] ${ }^{8}$

obteniéndose un valor de: $\mathrm{c}=346,44 \mathrm{~m} / \mathrm{s}$

${ }^{7}$ Todas las frecuencias registradas son menores a la frecuencia de corte (1)

${ }^{8}$ En la misma revista (JASA) aparecen otros modelos que podrían dar valores ligeramente diferentes. 
En la quinta columna se representa la relación entre la frecuencia de los modos con la frecuencia fundamental medidas experimentalmente. Se puede apreciar un corrimiento sistemático de los valores teóricos $L_{f}=L+0,6133 a(8)$ debido a la variación de la velocidad del sonido con la frecuencia. La última columna, columna 6, $\left(\mathrm{r}_{\mathrm{v}}\right)$ asegura la validez de la ecuación (16) para el cálculo de la velocidad de fase $\left(\mathrm{r}_{\mathrm{v}}>>1\right)$ y muestra lo pequeño del espesor de la capa límite viscosa $\left(\delta_{v}=0,19 \mathrm{~mm}\right.$ para $f_{1}=70,27 \mathrm{~Hz}$ y $\delta_{v}=0,048 \mathrm{~mm}$ para $\left.f_{8}=1070,77 \mathrm{~Hz}\right)$

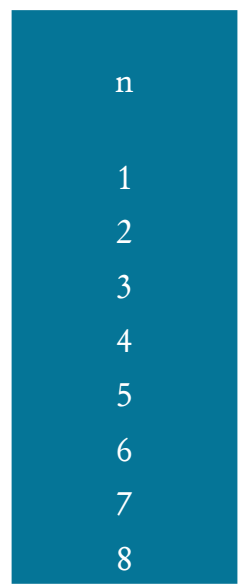

$\begin{array}{ccc}\mathrm{F}_{\mathrm{n}}(\mathrm{Hz}) & \mathrm{c}_{\mathrm{pex}}(\mathrm{m} / \mathrm{s}) & \mathrm{c}_{\mathrm{pteo}}(\mathrm{m} / \mathrm{s}) \\ 70,27(14) & 339,5(7) & 339,49 \\ 212,72(14) & 342,6(4) & 342,45 \\ 355,68(14) & 343,7(3) & 343,35 \\ 498,38(14) & 344,2(3) & 343,83 \\ 641,50(14) & 344,4(3) & 344,14 \\ 784,37(14) & 344,5(3) & 344,36 \\ 927,45(14) & 344,7(3) & 344,53 \\ 1070,77(14) & 344,9(3) & 344,66\end{array}$

$\frac{f_{n}}{f_{1} \exp }$

$r_{\mathrm{v}}$

1,000

52,0

3,027

90,5

5,062

117,1

7,092

138,6

9,129

157,2

11,162

173,9

13,198

189,1

15,238

203,1

Tabla 2: Frecuencias de resonancia, velocidad de fase experimental, velocidad de fase teórica, relación experimental entre la frecuencia de un modo y la frecuencia fundamental y relación entre el radio del tubo y el espesor de la capa límite viscosa para los distintos modos en un tubo abierto-cerrado.

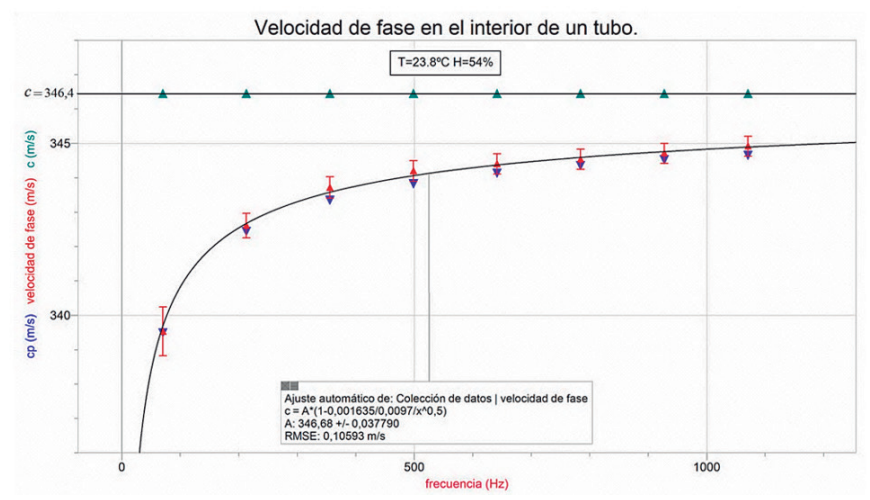

Fig. 5. Gráfica de la velocidad de fase en el interior del tubo para las frecuencias de los distintos modos normales (triángulo rojo) Con un triángulo azul, se representa la velocidad de fase del modelo teórico, ecuación (16), calculada usando la velocidad del sonido a esa temperatura y humedad y la curva continua el ajuste por mínimos cuadrados usando el modelo de la ecuación (16), en donde se dejó como parámetro libre la velocidad del sonido.

El gráfico de la figura 5 muestra que la velocidad del sonido en el interior de un tubo (velocidad de fase) es función de la frecuencia de las ondas estacionarias, por lo tanto, son ondas dispersivas, mostrando un excelente ajuste entre los valores experimentales y los valores teóricos, usando la ecuación (16) deducida por A. H Benade (Benade,1968).

Finalmente mediante el ajuste por mínimos cuadrados (dejando como parámetro libre c) se obtuvo el valor experimental para la velocidad del sonido de valor que presenta una desviación de $0,075 \%$ del valor aceptado a esa temperatura y humedad. 


\section{Validación del método}

Para validar el método anterior, que consiste en realizar un ajuste por mínimos cuadrados de la velocidad de fase experimental en función de la frecuencia:

$$
\mathrm{cp}=\mathrm{cp}(\mathbf{f})
$$

con el modelo de la ecuación (16) y dejando como parámetro libre la velocidad del sonido en el aire c, se procedió a tomar una serie de medidas a la misma temperatura $\mathrm{t}=28,3^{\circ} \mathrm{C}$ y humedad $\mathrm{H}=68 \%$ (ver tabla 3 ) usando 4 tubos de PVC. Los resultados se resumen en la tabla 3.

Tabla 3: Medida experimental de la velocidad del sonido en iguales condiciones de temperatura y humedad.

\begin{tabular}{ccccc}
$\begin{array}{c}\text { Tubo } \\
\text { PVC }\end{array}$ & Largo L $(\mathrm{m})$ & Radio a $(\mathrm{cm})$ & $\begin{array}{c}\text { velocidad del sonido } \\
\mathrm{c}(\mathrm{m} / \mathrm{s})\end{array}$ & $\begin{array}{c}\text { Desviación } \\
\text { Porcentual } \\
(\%)\end{array}$ \\
\hline 1 & $0,638(1)$ & $1,670(25)$ & $349,7(6)$ & 0,020 \\
2 & $0,850(1)$ & $1,670(25)$ & $349,7(6)$ & 0,023 \\
3 & $0,909(1)$ & $1,785(25)$ & $350,0(6)$ & 0,13 \\
4 & $1,880(1)$ & $2,805(25)$ & $349,4(6)$ & $-0,054$
\end{tabular}

El valor de la velocidad del sonido para esa temperatura y humedad de acuerdo con [12] es:

$$
\mathrm{c}=349,60 \mathrm{~m} / \mathrm{s}
$$

Todos los valores de la tabla 3, presentan una desviación muy pequeña respecto del valor aceptado, confirmando entonces el método utilizado.

\section{Conclusiones}

La velocidad del sonido en el aire se puede hallar usando el fenómeno de resonancia en tubos si se tiene un conocimiento exacto de como varía la velocidad de fase en función de la frecuencia en el interior de un tubo. Usualmente el efecto producido por las pérdidas viscosas y térmicas no es tenido en cuenta y se supone erróneamente que la velocidad con la que se propagan las ondas acústicas en el interior de un tubo (velocidad de fase) coincide con la velocidad del sonido (Cros, 2011). Ajustando la curva experimental a partir del modelo teórico dado por la ecuación (16) es posible determinar con gran precisión la velocidad del sonido c en el aire a una temperatura y humedad dadas.

Las medidas de la frecuencia de los modos de las ondas acústicas en el interior del tubo no requieren material de elevado costo (usando aplicaciones de análisis de sonido disponibles en teléfonos inteligentes) ni montajes complejos, por lo cual es adecuado para usar con estudiantes de formación de profesorado o estudiantes de grado universitarios de las áreas de ciencias o ingeniería. El análisis de los datos se puede realizar usando software de ajuste de curvas (Logger Pro de Vernier (Vernier) o aplicaciones disponibles para teléfonos, como Vernier graphicalanalysis(Vernier GraphicalAnalysis), Sparkvue Pasco (Sparkvue Par- 
co), etc. En la experiencia realizada en diferentes condiciones de temperatura y humedad se obtuvieron valores para la velocidad del sonido en el aire con una desviación máxima de $0,13 \%$ (una desviación menor a $0,50 \mathrm{~m} / \mathrm{s}$ para una velocidad del sonido de $346,4 \mathrm{~m} / \mathrm{s}$ ).

\section{Referencias}

BenadeA. H. (1968) "On the propagation of sound waves in a cylindrical conduit,'J. Acoust. Soc. Am. 44, 616-623

Cramer O. (1993). JASA, 93, p.2510.

Cros, Ana \& Ferrer Roca, Chantal. (2011). Física por un tubo. Mide la velocidad del sonido en el aire y diviértete con los tubos sonoros. Revista Eureka sobre enseñanza y divulgación de las ciencias. 8. 393-398. 10.25267/Rev_ Eureka_ensen_divulg_cienc.2011.v8.iextra.03.

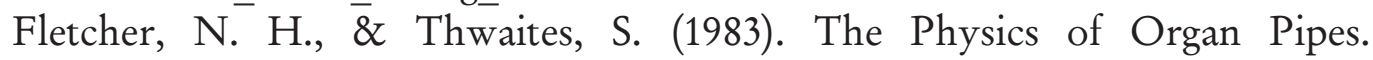 Scientific American, 248, 94 - 103. https://doi.org/10.1038/ SCIENTIFICAMERICAN0183-94

Gil S. (2014) Experimentos de Física usando TIC y elementos de bajo costo. Buenos Aires. Alfaomega.

Helmholtz H.v. (1863) Verhandlungen der Naturhistorisch Medizinischen Vereins zu Heidelberg, Bd III, 16.

KinslerL.E., A.R. Frey, A.B.C. Coppens and J.V. Sanders,(1995)Fundamentos de Acústica (Ed. Limusa, México

KirchhoffG. (1868)Poggendorfer Annalen 134,177-193. Ueber den Einfluss der Wärmeleitung in einem Gase auf die Schallbewegung.

Práctica de Ondas Mecánicas. Facultad de Ingeniería Cátedra de Física 1 (62.01) Ondas Mecánicas . Recuperado de: https://campus.fi.uba.ar/pluginfile. $\mathrm{php} / 231656 / \mathrm{mod}$ resource/content/0/1TPReson1C07.pdf

RayleighLord(1896)Theory of Sound Volume II.London. The Macmillan Company, second edition, 319-326.

RossingT. D. and N. H. Fletcher (1994), Principles of Vibration and Sound (Springer-Verlag, New York, sec. 8.2.

Sparkvue Parco. Google Play Store.Recuperado de: https://play.google.com/ store/apps/details? id =com.isbx.pasco.Spark\&hl=es_419

Spectrum Analyser.

Recuperado de: http://www.keuwl.com/SpectrumAnalyser/

Speed of sound in humid air ( Relative humidity).

Recuperado de http://www.sengpielaudio.com/calculator-airpressure.htm

Tijdeman H. (1975). On the propagation of sound waves in cylindrical tubes Journal of Sound and Vibration,39(8), p. 1-33.

Vernier. Recuperado de https://www.vernier.com/products/software/lp/

Vernier GraphicalAnalysis. Recuperado de: https://play.google.com/store/ apps/details? id=com.vernier.android.graphicalanalysis 